\title{
Exploring Markers of Exhausted CD8 T Cells to Predict Response to Immune Checkpoint Inhibitor Therapy for Hepatocellular Carcinoma
}

\author{
Chia-Lang Hsu a, b, c Da-Liang Ou ${ }^{b} \quad$ Li-Yuan Bai $^{d} \quad$ Chia-Wei Chen ${ }^{b} \quad$ Li Lin $^{b}$ \\ Shiu-Feng Huange Ann-Lii Cheng ${ }^{f, g, h}$ Yung-Ming Jeng ${ }^{i}$ Chiun Hsu ${ }^{b, f, g}$ \\ ${ }^{a}$ Department of Medical Research, National Taiwan University Hospital, Taipei, Taiwan; ${ }^{b}$ Graduate Institute of \\ Oncology, National Taiwan University College of Medicine, Taipei, Taiwan; ' Craduate Institute of Medical Genomics \\ and Proteomics, National Taiwan University College of Medicine, Taipei, Taiwan; ${ }^{d}$ Division of Hematology and \\ Oncology, Department of Internal Medicine, China Medical University Hospital and China Medical University, \\ Taichung, Taiwan; 'Institute of Molecular and Genomic Medicine, National Health Research Institutes, Miaoli, \\ Taiwan; ${ }^{f}$ National Taiwan University Cancer Center, Taipei, Taiwan; 9Department of Oncology, National Taiwan \\ University Hospital, Taipei, Taiwan; hepartment of Internal Medicine, National Taiwan University Hospital, Taipei, \\ Taiwan; 'Department of Pathology, National Taiwan University Hospital, Taipei, Taiwan
}

\section{Keywords}

T-cell exhaustion · Immune checkpoint inhibitor · Anti-PD-1 · Anti-PD-L1 - Tumor microenvironment

\begin{abstract}
Background: Reversal of CD8 T-cell exhaustion was considered a major antitumor mechanism of anti-programmed cell death-1 (PD-1)/ anti-programmed death ligand-1 (PD-L1)based immune checkpoint inhibitor $(\mathrm{ICI})$ therapy. Objectives: The aim of this study was to identify markers of T-cell exhaustion that is best associated with $\mathrm{ICl}$ treatment efficacy for advanced hepatocellular carcinoma (HCC). Methods: Immune cell composition of archival tumor samples was analyzed by transcriptomic analysis and multiplex immunofluorescence staining. Results: HCC patients with objective response after anti-PD-1/anti-PD-L1-based ICI therapy $(n=42)$ had higher expression of genes related to T-cell exhaustion. A 9-gene signature (LAG3, CD244, CCL5, CXCL9, CXCL13, MSR1, CSF3R, CYBB, and KLRK1) was defined, whose expression was higher in patients with response to ICI therapy, correlated with density of $\mathrm{CD} 8^{+} \mathrm{LAG}^{+}$cells in tumor microenvi-
\end{abstract}

ronment, and independently predicted better progressionfree and overall survival. This 9-gene signature had similar predictive values for patients who received single-agent or combination $\mathrm{ICl}$ therapy and was not associated with prognosis in HCC patients who received surgery, suggesting that it may outperform other T-cell signatures for predicting efficacy of $\mathrm{ICl}$ therapy for HCC. For HCC patients who underwent surgery for both the primary liver and metastatic lung tumors ( $n=31$ ), lung metastatic HCC was associated with a higher exhausted CD8 T-cell signature, consistent with prior observation that patients with lung metastatic HCC may have higher probability of response to ICI therapy. Conclusions: CD8 T-cell exhaustion in tumor microenvironment may predict better efficacy of ICI therapy for HCC.

(C) 2021 The Author(s)

Published by S. Karger AG, Basel

Chia-Lang Hsu and Da-Liang Ou contributed equally to this work. Dr. Da-Liang Ou is a National Taiwan University YongLin Scholar since 2018.

Part of this study has been presented at the International Liver Congress $^{\mathrm{TM}}$ 2019, 10-14 April 2019, Vienna, Austria.
(C) 2021 The Author(s)

Published by S. Karger AG, Basel

This is an Open Access article licensed under the Creative Commons Attribution-NonCommercial-4.0 International License (CC BY-NC) (http://www.karger.com/Services/OpenAccessLicense), applicable to the online version of the article only. Usage and distribution for commercial purposes requires written permission.
Correspondence to:

Yung-Ming Jeng, mrna0912@gmail.com

Chiun Hsu, chsu1967@ ntu.edu.tw 


\section{Introduction}

Immune checkpoint inhibitor (ICI) therapy targeting the programmed cell death-1 (PD-1)/programmed death ligand-1 (PD-L1) pathway has been approved for the treatment of more than 10 cancer types [1,2]. For patients with advanced hepatocellular carcinoma (HCC), the antiPD-1 agents' nivolumab and pembrolizumab demonstrated an objective response rate (ORR) of approximately $15 \%$ and median overall survival (OS) of about 1 year in patients who had prior treatment with sorafenib $[3,4]$. Unfortunately, this initial promising antitumor activity of anti-PD-1 ICI therapy was not translated into OS benefit when compared with sorafenib as first-line therapy [5] or with placebo as second-line therapy [6].

The most extensively studied biomarkers for antiPD-1 ICI therapy include tumor mutation burden (TMB), expression of PD-L1 in tumor or immune cells, and expression patterns of immune-related genes in the tumor microenvironment [7-9]. However, the predictive value of TMB and PD-L1 expression for HCC was difficult to establish because of the low incidence of high TMB or high PD-L1 expression in HCC tumors [10, 11]. Immunerelated gene signatures were recently shown to correlate with objective tumor response and survival in HCC patients who received nivolumab therapy [12]. The clinical utility and the biological implications of specific genes in these signatures are worthy of further exploration.

Reversal of CD8 T-cell exhaustion was considered a major antitumor mechanism of ICI therapy $[13,14]$. The concept of CD8 T-cell exhaustion was originally established primarily by studies using models of chronic viral infection [15-17]. Cancer-induced T-cell exhaustion shares the common features of chronic antigen exposure as viral infection, but additional confounding factors, such as tumor-intrinsic genetic and epigenetic abnormalities and immune suppression induced by prior anticancer therapy, should be considered in defining the biomarkers of cancer-related T-cell exhaustion [18].

In this study, we analyzed the immune cell composition of the archival HCC tumor samples from the HCC patients who received anti-PD-1/anti-PD-L1-based ICI therapy by using multiplex immunofluorescence staining and transcriptomic analysis of immune-related genes, and markers of CD8 T-cell exhaustion were found to be associated with superior treatment efficacy. Tumor samples from additional cohorts of HCC patients who underwent surgery were analyzed to explore the potential prognostic value of $\mathrm{T}$-cell exhaustion biomarkers and the validity of using archival tumor tissue for the prediction of treatment efficacy.

\section{Materials and Methods}

\section{Patients}

This study was approved by the Research Ethics Committee, National Taiwan University Hospital (Approval Nos. 201308035RINC and 201706007RINA). Archival tumor tissues obtained from 2 cohorts of HCC patients were analyzed for potential predictors of response to anti-PD-1/anti-PD-L1 therapy.

The first cohort consisted of patients who underwent antiPD-1/anti-PD-L1-based therapy at National Taiwan University Hospital (the ICI therapy cohort). Archival tumor tissue from prior surgery with curative intent or excisional biopsy of metastatic lesions before the start of ICI therapy was obtained for biomarker research, and all the patients signed written informed consent for this study. Treatment response was evaluated by imaging according to Response Evaluation Criteria in Solid Tumors 1.1. The second cohort consisted of HCC patients who had received surgery for both the primary liver and the metastatic lung tumors at NTUH and was used to explore the potential difference in the tumor immune microenvironment (the primary-metastasis cohort).

Transcriptomic Analysis of Immune-Related Genes

The sections of the formalin-fixed, paraffin-embedded (FFPE) tissue $(10-\mu \mathrm{m}$ thick) were first examined by the pathologist (Y.M.J.) to determine the tumor area. Total tumor RNA was extracted using the RNeasy FFPE Kit (Qiagen, Valencia, CA, USA) and hybridized to nCounter ${ }^{\circledR}$ probes for the 770 predefined genes of the PanCancer Immune Profiling Panel (NanoString Technologies Inc., Seattle, WA, USA). Gene counts for each individual sample were normalized by the trimmed mean of $M$-values method [19] and quantile normalization and then converted to log-transformed counts per million for further analysis. Immune cell composition of each sample was calculated as the average expression of the corresponding marker genes of each immune cell type, as defined by Danaher et al. [20]. The published immune-related signatures that may help predict response to ICI therapy in various cancers were used to assess the prediction ability of ICI treatment response in HCC $[9,12,20-26]$ (detailed description and methods listed in the online suppl. Methods and Materials; for all online suppl. material, see www.karger.com/doi/10.1159/000515305).

\section{Multiplex Immunofluorescence Staining}

The FFPE tissue sections were studied by using the Polaris ${ }^{\circledR}$ system (PerkinElmer, Waltham, MA, USA) with the Opal ${ }^{\mathrm{TM}} 7$ Solid Tumor Immunology Kit (OP7TL4001KT) or the customized Opal 4-color panel according to the manufacturer's instructions. The Opal 4-Color anti-rabbit automation IHC Kit (NEL830001KT) was used to measure LAG3 and PD1 expression that was associated with CD8 T-cell exhaustion (see online suppl. Fig. 1; online suppl. Table 1 for detailed procedures).

\section{External Data of HCC Gene Expression Patterns}

The Cancer Genome Atlas Liver HCC (TCGA-LIHC) RNAseq and clinical data were fetched via the Genome Data Commons data portal. Gene-level read counts across all the samples were normalized using the Trimmed Mean of M-values method [19]. OS and progression-free survival (PFS) data from the TCGA-LIHC were collected and standardized as previously described [27]. The immune subtypes of TCGA-LIHC were defined by previous study [28]. 
Comparison of Immune Profiles between Primary and

Metastatic HCC

Multiplex immunofluorescence staining and transcriptomic analysis using the Nanostring method were performed for FFPE tumor samples from the primary-metastasis cohort. The similarity of immune cell compositions between the matched primary and metastatic tumors was determined by calculating the Pearson correlation coefficient. The background distribution of the immune cell composition similarity for the matched primary-metastatic tumors was estimated by shuffling the relationships of the matched primary-metastatic tumors. To compare the distribution of similarity in the matched normal liver and lung tissues, the RNA-seq datasets from the 192 normal liver-lung tissue pairs were obtained from the Genotype-Tissue Expression data portal. The gene-level read counts from Genotype-Tissue Expression were normalized as described above.

\section{Statistical Analysis}

The Kaplan-Meier curves were used to assess the correlation between immune cell abundance/score and survival (OS and PFS). The samples were stratified into 2 groups based on the median value of the given features, and the log-rank test was used to assess the difference. Hazard ratios of various immune cell abundance and scores for OS and PFS without/with adjustment of clinicalpathological variables were estimated by the Cox proportional hazards model. For the Cox model, each immune cell abundance or score was standardized to have a mean of 0 and a standard deviation of 1 . To avoid the overfitting of the models, the clinicalpathological characteristics with frequency $>20 \%$ of the given cohort were considered in the multivariate analyses. All of the analyses mentioned above were performed in the R language ( $v$ 3.5.2).

Data Availability

The raw and processed data of the IO cohort and the primarymetastatic cohort are all available in GEO with accession numbers GSE140901 and GSE141016, respectively.

\section{Results}

Prediction of ICI Efficacy by Transcriptomic Analysis: Characterization of an "Exhausted" CD8 Signature

Sixty-one patients with HCC who were treated with anti-PD1/anti-PD-L1-based therapy at National Taiwan University Hospital whose FFPE tumor tissues were available were identified (online suppl. Tables 2,

Fig. 1. Exhausted CD8 T-cell signature may predict efficacy of ICI therapy. a Flow chart of the study sample enrollment. b Heatmap visualizing the immune cell composition of HCC tumors from the ICI therapy discovery cohort $(n=24)$. c PCA of the immune cell composition across all tumors. The dot plot showed the variance explained by the 2 principal components (PC1 and PC2). d The bar plot showed the contribution of each immune cell type to the PC2 of PCA in (c). e Bar plot depicting AUC for the prediction of objective response by different immune cells. The performance of
3). Only 42 tumors from these patients fulfilled the assessment of sample quality, and the gene expression was profiled by the NanoString nCounter platform. To assess the robustness of the identified signatures or biomarkers, the samples were separated into 2 groups based on the date of enrollment: one for biomarker discovery (discovery cohort, $n=24$ ) and the other for validating the finding (validation cohort, $n=18$ ) (Fig. 1a; online suppl. Tables 2,3). Fewer patients in the validation cohort had macrovascular invasion and prior systemic therapy, which may partly explain the reason that the validation cohort had better PFS and OS (online suppl. Table 2; online suppl. Fig. 2). No other significant difference between the discovery and validation cohorts in terms of clinical-pathological characteristics was found. The median intervals from the obtainment of tumor samples (from prior surgery or excisional biopsy) to the start of ICI-based therapy were 1.3 years (range 0-12.0 years) for the discovery cohort and 0.7 years (range 0-4.4 years) for the validation cohort, respectively ( $p=0.13$ by Student's $t$ test). The ORR of the ICI therapy cohort was $37.7 \%$, and there were no clinical-pathological characteristics associated with ORR, PFS, or OS (online suppl. Tables 4, 5). Although the ORR appeared higher than other trials, more balanced numbers of responders and nonresponders for analysis provided better statistical power for biomarker discovery.

The best radiographic responses in the discovery cohort were 6 partial responses (PR), 10 stable diseases $(\mathrm{SD})$, and 8 progressive diseases (PD). Principal-component analysis of the immune cell composition, measured by gene expression patterns, indicated distinct expression patterns between ICI responders and non-responders (Fig. 1b), and the second principal component accounted for the major difference (Fig. 1c). The exhausted CD8 T cells (defined by the expression of LAG3, CD244, and EOMES) were the major cell type accounting for the variations in second principal component (Fig. 1d), and the exhausted CD8 T-cell signature had the best performance

a random predictor $(\mathrm{AUC}=0.5)$ was denoted by the dashed line. f Forest plots showing hazard ratios of various immune cells for progress-free survival (PFS, left) and OS (right). Horizontal bars represented the 95\% CIs of HR. g PFS and OS curves for the 24 HCC patients stratified by the exhausted CD 8 T cells. ICI, immune checkpoint inhibitor; HCC, hepatocellular carcinoma; OS, overall survival; PFS, progression-free survival; PC2, second principal component; PCA, principal-component analysis; ROC, receiveroperating characteristics; AUC, area under the ROC curve.

(For figure see next page.) 
a

\begin{tabular}{|}
$\begin{array}{r}\text { Assessed for eligibility } \\
(\mathrm{n}=61)\end{array}$ \\
\hline \begin{tabular}{c|c|} 
RNA quality assessment \\
$(\mathrm{n}=42)$
\end{tabular} \\
\hline $\begin{array}{c}\text { Discovery } \\
\text { cohort }\end{array}$ & $\begin{array}{c}\text { Validation } \\
\text { cohort } \\
\text { (n=24) }\end{array}$ \\
\hline
\end{tabular}

b

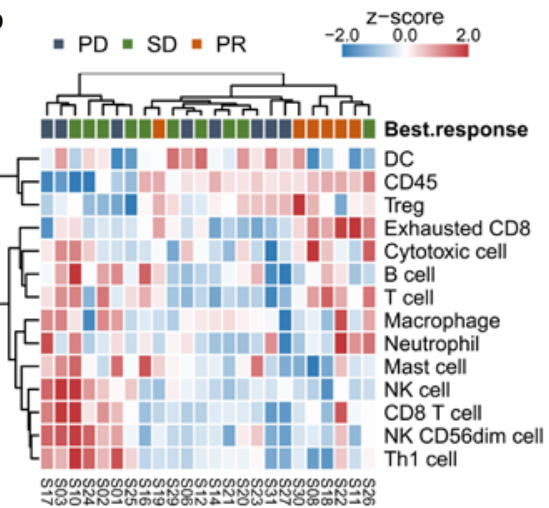

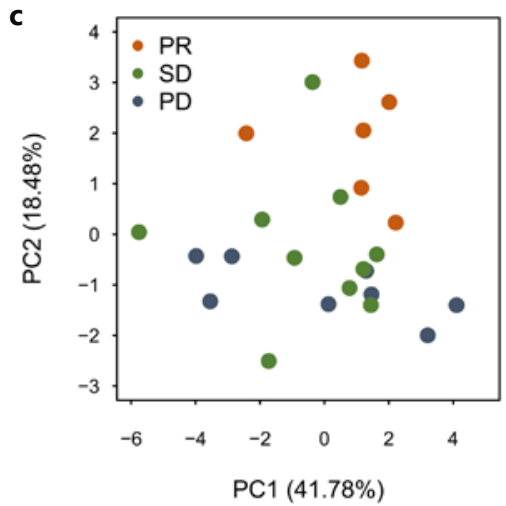

d
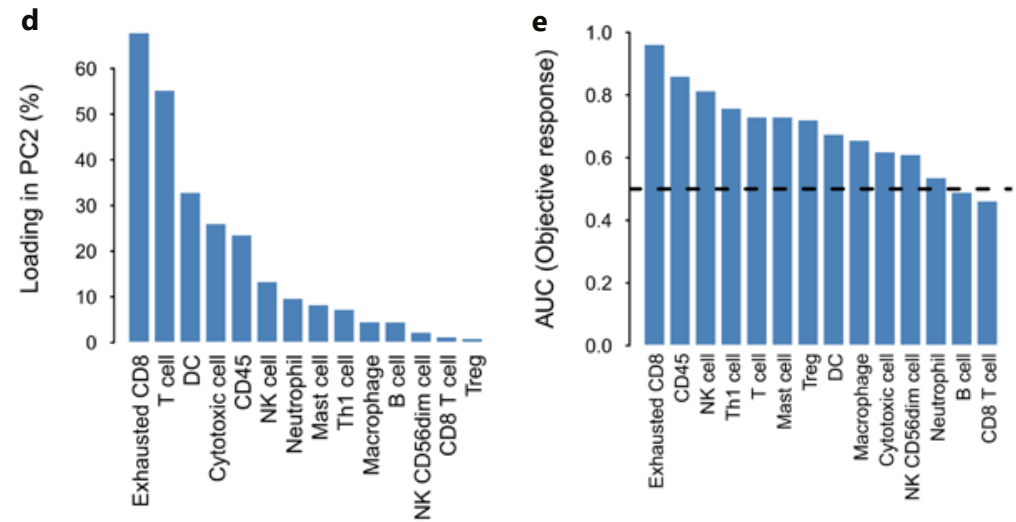

f

PFS
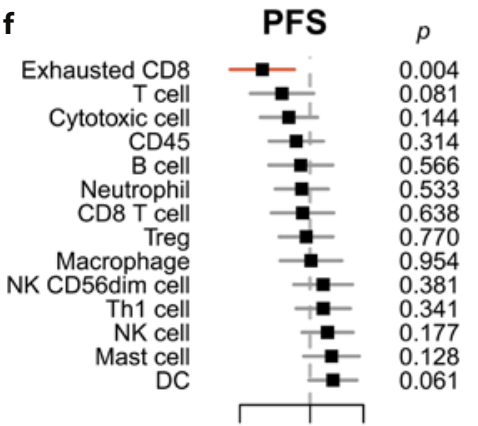

0.004
0.081

0.081
0.144

0.314
0.566

0.566
0.533

0.638
0.770

0.954

0.381

0.341

0.177
0.128

0.061

$\begin{array}{lll}0.3 & 1.0 & 2.5\end{array}$

Hazard Ratio

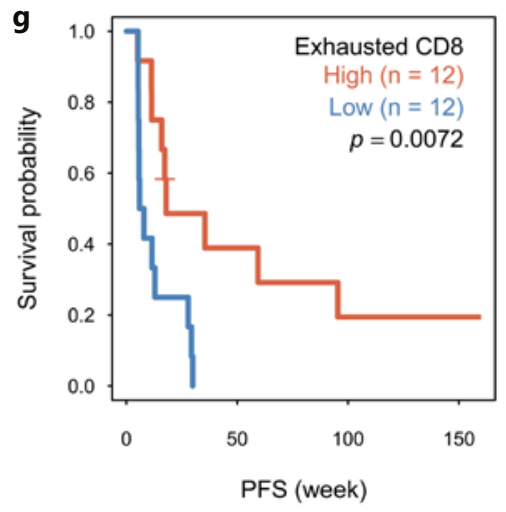

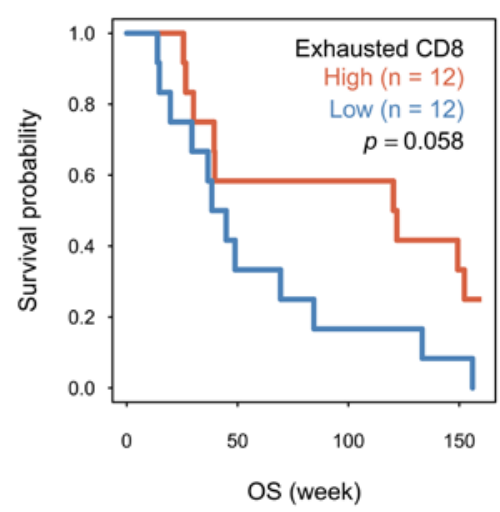

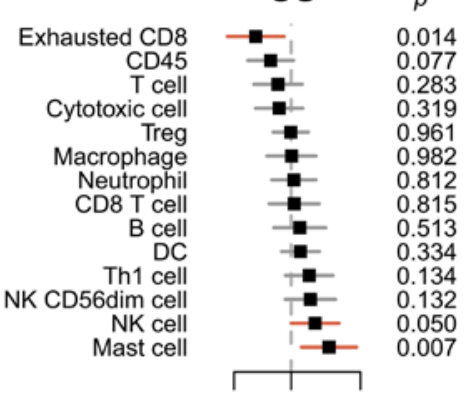

$\begin{array}{lll}0.3 & 1.0 & 4.3\end{array}$

Hazard Ratio 


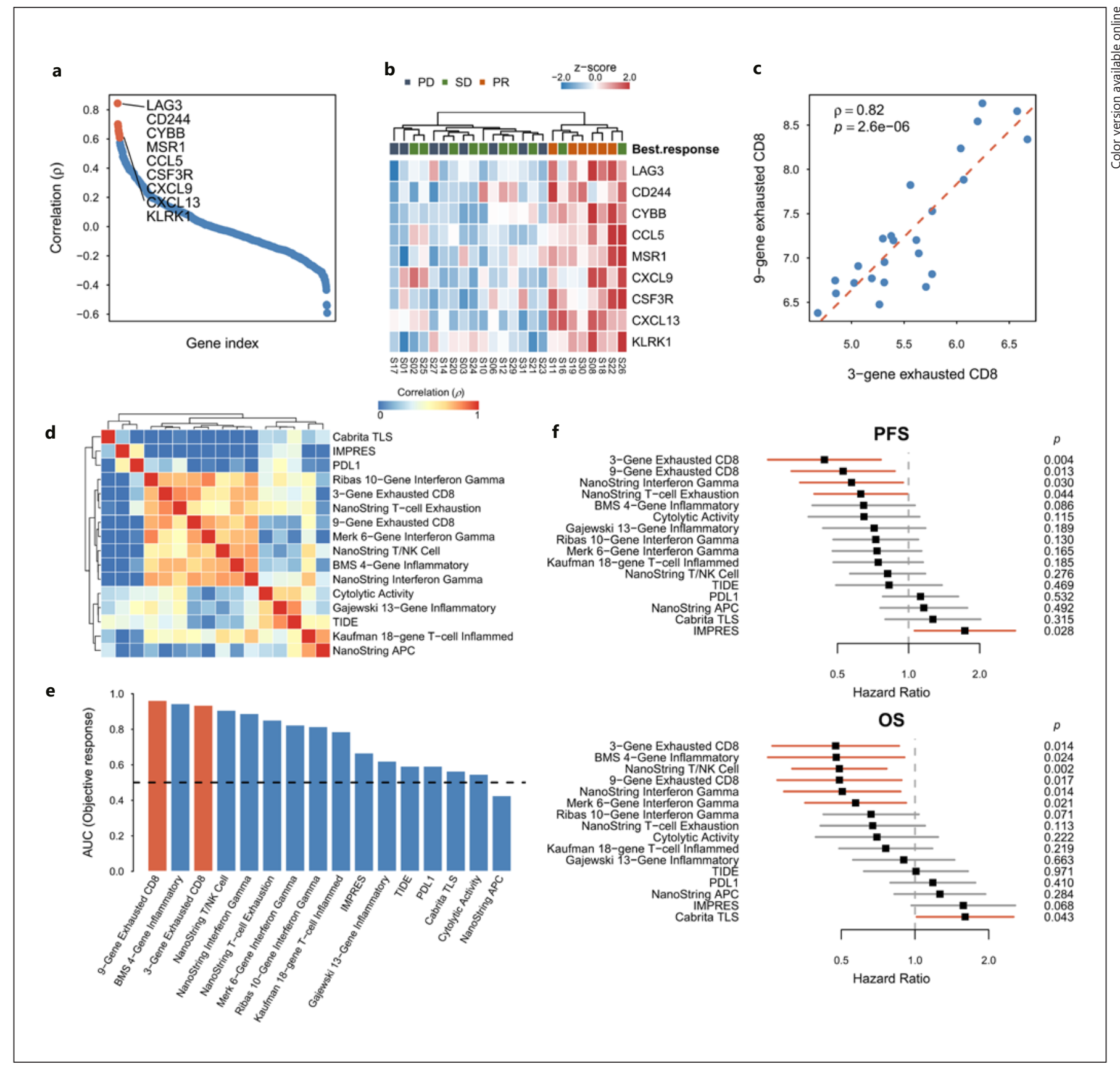

Fig. 2. Exploration of genes associated with CD8 T-cell exhaustion. a Correlation between the 3-gene exhausted CD8 signature (LAG3, CD244, and EOMES) and expression of other immune-related genes in the Nanostring panel. Genes with the Spearman's correlation coefficient $(\rho)>0.6$ were highlighted in red and labeled. $\mathbf{b}$ Expression profiles of the 9 genes associated with the exhausted CD8 T-cell signatures cross the 24 HCC tumors. c Scatter plot between the 3-gene and the 9-gene exhausted CD8 T-cell signatures in individual HCC tumors (Spearman's correlation coefficient $\rho=$
0.82). d Correlation matrix among representative T-cell-related signatures and signatures associated with response to ICI therapy. e The AUC for predicting objective response in the discovery cohort of HCC patients $(n=24)$ who received ICI therapy. The performance of a random predictor (AUC $=0.5$ ) was represented by the dashed line. $\mathbf{f}$ The forest plots of HR for PFS (upper panel) and OS (lower panel) determined by various signatures. Horizontal bars represented the 95\% CIs of HR. HCC, hepatocellular carcinoma; OS, overall survival; PFS, progression-free survival. 
a

\begin{tabular}{|c|c|c|c|c|}
\hline \multirow[t]{2}{*}{ Characteristics } & \multicolumn{2}{|c|}{ Progress-free survival } & \multicolumn{2}{|l|}{ Overall survival } \\
\hline & HR $(95 \%$ IC) & $p$ & HR $(95 \%$ IC) & $p$ \\
\hline$N=24$ & Event $=21$ & & Event $=21$ & \\
\hline 9-gene exhausted CD8 & $0.29(0.13-0.68)$ & 0.0038 & $0.21(0.08-0.55)$ & 0.0015 \\
\hline \multicolumn{5}{|l|}{ Age } \\
\hline$\leqq 60$ & Ref & & Ref & \\
\hline$>60$ & $1.85(0.66-5.16)$ & 0.24 & $3.67(1.16-11.6)$ & 0.027 \\
\hline \multicolumn{5}{|l|}{ ALBI grade } \\
\hline 1 & Ref & & Ref & \\
\hline 2 & $0.34(0.09-1.30)$ & 0.12 & $0.28(0.07-1.05)$ & 0.058 \\
\hline \multicolumn{5}{|l|}{ NLR } \\
\hline$\leqq 3$ & Ref & & Ref & \\
\hline$>3$ & $0.47(0.16-1.42)$ & 0.18 & $0.97(0.28-3.30)$ & 0.96 \\
\hline \multicolumn{5}{|l|}{ AFP (ng/ml) } \\
\hline$\leqq 100$ & Ref & & Ref & \\
\hline$>100$ & $6.50(1.51-28.1)$ & 0.012 & $6.53(1.63-26.2)$ & 0.008 \\
\hline \multicolumn{5}{|l|}{ Macrovascular invasion } \\
\hline No & Ref & & Ref & \\
\hline Yes & $0.76(0.24-2.46)$ & 0.65 & $1.22(0.39-3.84)$ & 0.73 \\
\hline
\end{tabular}

b

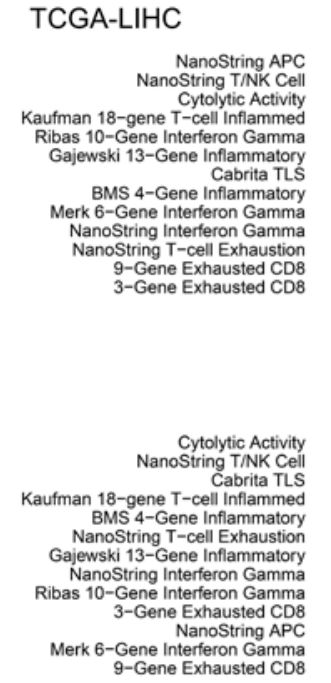

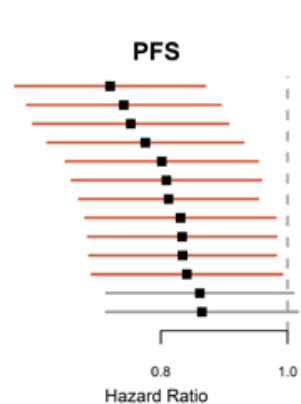

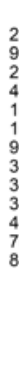

OS

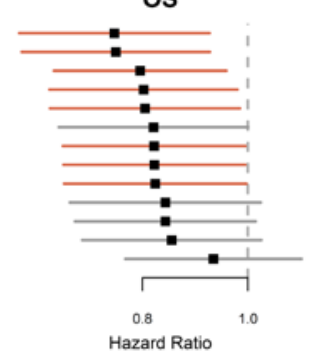

Fig. 3. Predictive versus prognostic value of exhausted CD8 T-cell signatures. a Multivariate analysis for PFS and OS in the discovery cohort $(n=24)$. b Forest plots showing hazard ratios of various immune-related signatures for PFS (upper panel) and OS (lower panel) in the TCGA-LIHC. Horizontal bars represented the $95 \%$ CIs of HR. OS, overall survival; PFS, progression-free survival. with AUC of 0.96 for predicting objective response (Fig. 1e). Patients with highly exhausted CD8 T cell had significantly better PFS and OS (Fig. 1f, g).

We further identified 9 genes in the Nanostring panel whose expression correlated highly with the exhausted CD8 T-cell signature (Spearman's correlation coefficient $\rho>0.6$, Fig. 2a, b). These genes regulated cytokine expression (CCL5, CXCL9, and CXCL13), functions of macrophages (MSR1 and CSF3R) or neutrophil (CYBB), and activation of T cells or NK cells (KLRK1). Expression of this expanded 9-gene signature correlated well with the original 3-gene exhausted CD8 T-cell of our signature (Fig. 2c). Correlation exhausted CD8 T-cell signatures was systematically compared with 11 signatures that might be associated with response to ICI therapy and 3 ICI response predictive scores (the gene list was summarized in online suppl. Methods and Materials) (Fig. 2d). A high correlation between our exhausted CD8 T-cell signatures was found with the interferon- $\gamma$ signatures, a previously reported $\mathrm{T}$ cell exhaustion signature [20], and the Bristol-MyersSquibb (BMS) 4-gene inflammatory signature [12]. On the other hand, the previously defined ICI response scores were poorly correlated with our exhausted CD8 T-cell signatures. The performance of the expanded 9-gene ex- hausted CD8 T-cell signature in predicting objective response to ICI therapy, PFS, and OS was among the best in the signatures examined (Fig. 2e, f). Bivariate and multivariate analyses with other clinicopathological factors indicated that the predictive value of the 9-gene exhausted CD8 T-cell signature remained significant in most of the subgroups (Fig. 3a; online suppl. Table 6). Similar results were also observed in the validation cohort (online suppl. Fig. 3). Our data support the hypothesis that gene expression profiles reflecting the $\mathrm{T}$-cell functional status in the tumor microenvironment may help predict response of HCC patients to ICI-based therapy.

To explore whether the expression patterns may also be of prognostic significance, regardless of the therapies the patients may receive, the gene expression data from the TCGA-LIHC database were used to analyze the potential prognostic impact of the signatures described above. The 9-gene exhausted CD8 T-cell signature that we established appeared the least associated with PFS or OS (Fig. 3b). The results indicated that in predicting efficacy of ICI therapy for HCC patients, our 9-gene exhausted CD8 T-cell signature may be less confounded by unknown immunological factors with potential prognostic significance. 


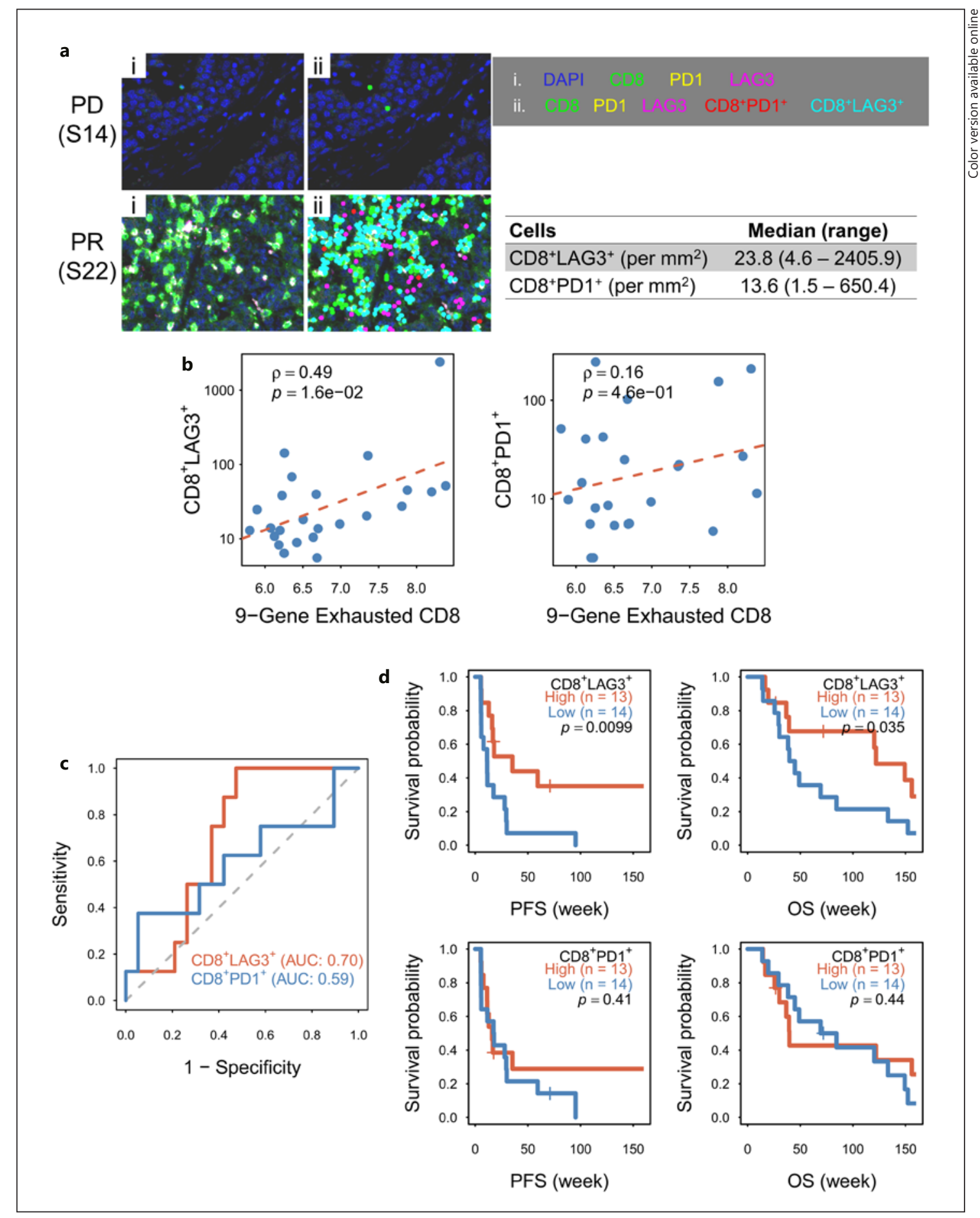

Fig. 4. Validation of exhausted CD8 infiltration using multiplex immunofluorescence staining. a Representative images $(\times 20$ magnification) from the tumors with low (top) and high (bottom) infiltrated exhausted CD8 T cells from patients with PD or PR from the ICI therapy cohort (right panel). Spectrally unmixed images (i); overlaid phenotyping images of each cell type (ii). b Correlation between the 9-gene exhausted CD8 T-cell signatures and densities of exhausted $\mathrm{CD} 8$ cells $\left(\mathrm{CD} 8^{+} \mathrm{LAG}^{+}\right.$or $\left.\mathrm{CD}^{+} \mathrm{PD}^{+}\right)$calculated by
Spearman correlation coefficient $(\rho)$ in the ICI therapy discovery cohort $(n=24)$. c The ROC curves for the densities of exhausted $\mathrm{CD} 8$ cells $\left(\mathrm{CD}^{+} \mathrm{LAG}^{+}\right.$or $\left.\mathrm{CD}^{+} \mathrm{PD}^{+}\right)$to predict objective response after ICI therapy. d The PFS and OS curves for patients in the ICI therapy discovery cohort, stratified by the cell density of exhausted $\mathrm{CD} 8\left(\mathrm{CD}^{+} \mathrm{LAG}^{+}\right.$or $\left.\mathrm{CD}^{+} \mathrm{PD}^{+}\right) \mathrm{T}$ cells. OS, overall survival; PFS, progression-free survival; PR, partial response; PD, progressive disease; ROC, receiver-operating characteristics. 
Correlation between the Exhausted CD8 T-Cell

Signature and the Expression of Exhausted CD8

T-Cell Markers in the Tumor Microenvironment

Expression of LAG3 and PD1 on CD8 T cells was measured by multiplex immunofluorescence staining in the discovery cohort (Fig. 4a). The exhausted CD8 T-cell signature correlated significantly with the density of $\mathrm{CD}^{+} \mathrm{LAG}^{+}$cells, but not $\mathrm{CD} 8^{+} \mathrm{PD} 1^{+}$cells (Fig. $4 \mathrm{~b}$ ). Higher density of $\mathrm{CD} 8^{+} \mathrm{LAG}^{+}$cells was associated with better objective response to ICI therapy and longer PFS, and OS (Fig. 4c, d). The above data support our hypothesis that the biomarkers of CD8 T-cell exhaustion may help predict efficacy of ICI therapy for HCC.

Association between Exhausted CD8 T-Cell Signature and WNT/ $\beta$-Catenin Pathway and Other Immune-

Based Classifications

Previous studies indicated that aberrant $W N T / \beta$ catenin signaling was associated with lower ORR and shorter PFS and OS after ICI therapy [29]. To examine the $\mathrm{WNT} / \beta$-catenin status of the ICI therapy cohort, we performed Sanger sequencing of CTNNB1 exon3 as well as $\beta$-catenin and glutamine synthetase immunostaining [30, 31] (online suppl. Fig. 4a). Thirty-four of 42 subjects had adequate tumor tissue for genomic DNA analysis. Only 1 HCC harbors CTNNB1 mutations and 4 and 6 HCCs had nuclear $\beta$-catenin and cytoplasmic glutamine synthetase expression, respectively (online suppl. Fig. 4b). Aberrant $\beta$-catenin status was not strongly associated with the treatment response to ICI, possibly because of the low number of subjects with aberrant $\beta$-catenin status in our cohort. This may result from the high proportion of subjects with chronic hepatitis B virus infection, which was associated with a low probability of aberrant $\beta$-catenin status in the tumors. We further examined the exhausted CD8 T-cell signature in TCGA-LIHC and found samples with CTNNB1 mutation have significantly lower exhausted CD8 scores (online suppl. Fig. 4c), consistent with the previous work suggesting that the aberrant $\mathrm{WNT} / \beta$-catenin pathway may prevent $\mathrm{T}$-cell infiltration in HCC $[22,32,33]$.

The immune-based subtypes of HCC have been comprehensively investigated $[28,33,34]$. To explore which types of immune cells resided in exhausted CD8 high samples, we utilized the CIBERSORT [35] for the deconvolution of the ICI therapy cohort. Although the overall number of TILs was not correlated with the 9-gene exhausted CD8 T-cell signature, abundance of T cells and macrophages was positively correlated with exhausted CD8 T cells (online suppl. Fig. 5a, b). Higher exhausted
CD8 signature was also correlated with the immune class HCC defined by Sia et al. [34] (online suppl. Fig. 5c). Finally, the 9-gene exhausted CD8 T-cell signatures were significantly enriched in the IFN- $\gamma$ dominant subtype of HCC in the TCGA-LIHC database, which was classified into 4 major immune subtypes, namely wound healing, IFN- $\gamma$ dominant, inflammatory, and lymphocyte depleted [28] (online suppl. Fig. 5d). Overall, our 9-gene exhausted CD8 T-cell signature was associated with high T-cell infiltration in HCC.

\section{Exhausted CD8 T-Cell Signature in ICI Single-Agent} and ICI-Based Combination Therapies

We asked whether the exhausted CD8 T-cell signatures have similar ability to predict efficacy of singleagent ICI therapy versus ICI-based combination therapies. In the ICI therapy cohort, 22 and 20 patients received ICI single-agent and ICI-based combination therapies, respectively. There was no significant difference in terms of clinicopathological features and survival between these 2 groups (Fig. 5a; online suppl. Table 7). In both the single-agent and combination therapy groups, tumors from responders had higher infiltration of exhausted CD8 T cells (Fig. 5b) and the 9-gene exhausted CD8 T-cell signature had better predictive ability for ORR (Fig. 5c, d), PFS, and OS (Fig. 5e, f; online suppl. Tables 8-11), compared with other reported signatures of immune-related gene expression.

Comparison of the Immune Tumor Microenvironment between the Primary and Metastatic HCC

Another clinically relevant issue is whether the composition of immune cells or expression of immune-related genes measured from tumor samples from different anatomical sites may differ and thus confound the analysis of predictive biomarkers. We measured the immune composition of the HCC tumor samples from the $31 \mathrm{pa}-$ tients who underwent surgery for both the primary HCC and metastatic HCC to lungs by both multiplex immunofluorescence staining and transcriptomic analysis to address this issue (see online suppl. Table 12 for the baseline characteristics). The immune cell composition measured by either method varied widely among the patients and did not seem to correlate with the duration between the resection of the primary and the metastatic tumors (Fig. 6a, b). The similarity of immune cell composition in the primary and metastatic tumors was quantified by the Pearson correlation coefficient. Compared with 500 random tumor pairs, the cell density and cell fraction measured by multiplex immunofluorescence staining and the 


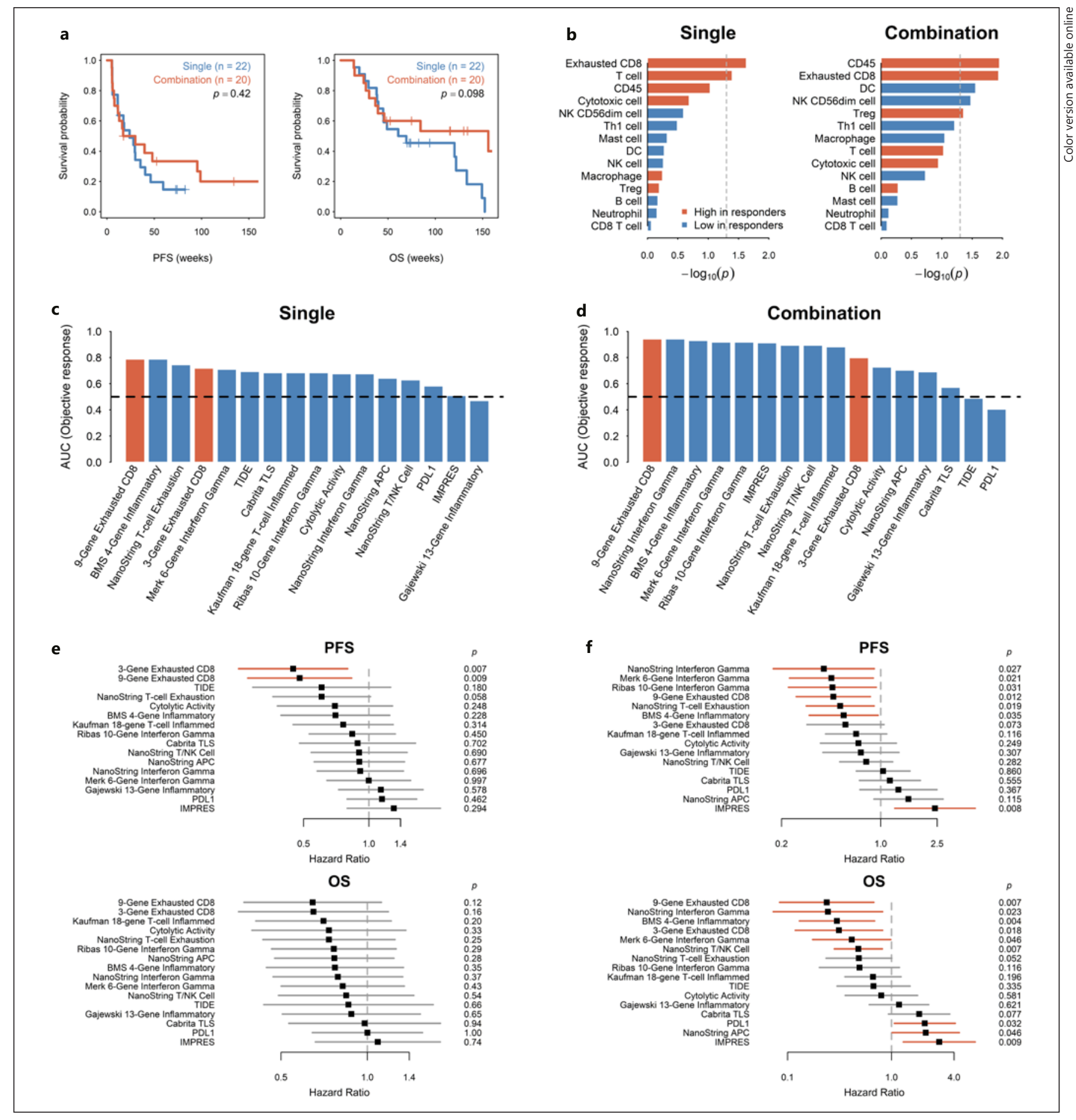

Fig. 5. Predictive ability of exhausted CD8 T-cell signatures for single-agent ICI and ICI-based combination therapy. a PFS and OS curves of patients with single-agent ICI and ICI-based combination therapies. $\mathbf{b}$ Bar plot depicting the statistical significance of each immune cell between the ICI responders and nonresponders. The red and blue represent the high and low abundance of the given immune cells in responders. The dash line is the $p=0.05$. c, d The AUC for predicting objective response in the ICI single- agent (c) and combination therapies (d). The performance of a random predictor $(\mathrm{AUC}=0.5)$ was represented by the dashed line. e, $\mathbf{f}$ Forest plots showing hazard ratios of various immune-related signatures for PFS (top) and OS (bottom) in the ICI single-agent (e) and combination therapies (f). Horizontal bars represented the 95\% CIs of HR. OS, overall survival; PFS, progression-free survival; AUC, area under ROC curve; ROC, receiver-operating characteristics. 


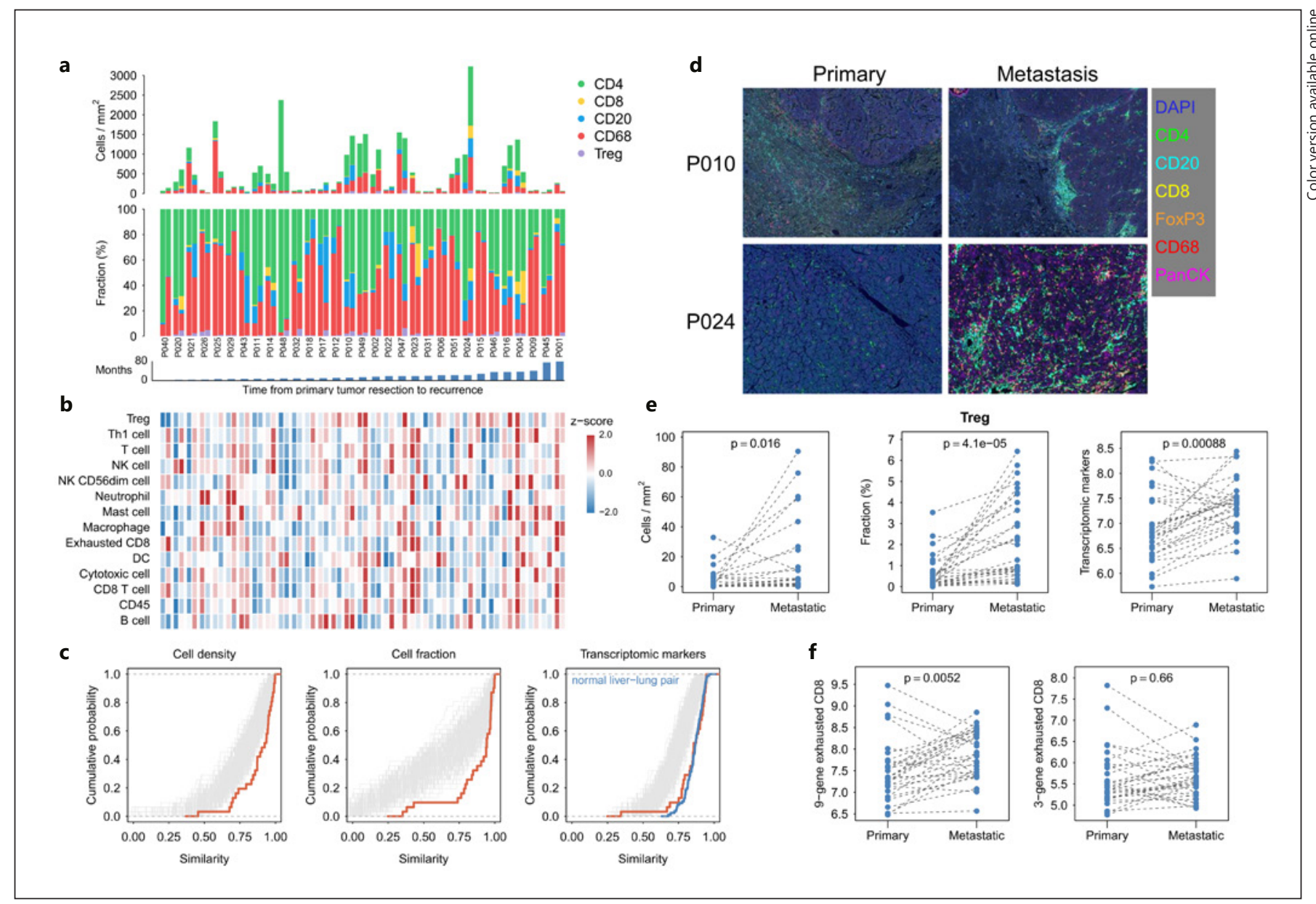

Fig. 6. Immune profiles of matched primary liver and metastatic lung HCC. a Immune cell composition (upper panel, cell density; lower panel, cell fraction) of the tumor pairs quantified by multiplex immunofluorescence staining. For each tumor pair, the left bar indicated primary tumor and the right bar indicated metastatic lung tumor. The patients are sorted based on the time interval between the primary tumor resection and recurrence. b Immune cell composition estimated by the transcriptomic markers. c Cumulative distributions of the immune cell composition similarities, determined by multiplex immunofluorescence staining (cell density and fraction) and transcriptomic analysis (Nanostring panel), between primary and metastatic tumors (red) and 500 random pairs (gray). The sim- ilarity is quantified by the Pearson correlation coefficient. d Representative phenotyping images ( $\times 20$ magnification) from the tumors with low (upper panel) and high (lower panel) immune heterogeneity between the primary and metastatic tumors. The HCC tumor samples were labeled with the Opal 7-Color Multiplexed IHC Kit. e Comparison of distribution of Treg cells between primary and metastatic HCC. Treg cells were determined by the positivity of CD4 and Foxp3 using multiplex immunofluorescence staining and by the transcriptomic expression of FOXP3, IL2RA, and MRC1 genes. $\mathbf{f}$ Comparison of the scores of the 9-gene exhausted CD8 T-cell signature between the primary and metastatic HCC. HCC, hepatocellular carcinoma; OS, overall survival. distribution of transcriptomic markers were very similar between the primary and metastatic tumors (Fig. 6c). Besides, the distribution of transcriptomic markers between the primary and metastatic tumors was similar to that of matched normal liver and lung tissues, suggesting that the immune cell composition is not related to tissue types (Fig. 6c). However, the overall similarity in immune cell composition between the primary and metastatic HCC did not preclude the possibility of variations in individual immune cells (Fig. 6d). For example, Treg cells (defined as $\mathrm{CD}^{+}{ }^{+} \mathrm{FoxP}_{3}{ }^{+}$cells) were significantly more abundant in metastatic HCC tumors (Fig. 6e). The scores of the 9-gene exhausted CD8 T-cell signature were also higher in metastatic HCC tumors (Fig. 6f). 


\section{Discussion/Conclusion}

In this study, we evaluated the transcriptomic and protein markers of T-cell exhaustion that may predict response to ICI therapy for HCC. We identified a signature consisting of $9 \mathrm{~T}$-cell exhaustion-related genes that were correlated with density of $\mathrm{CD}^{+} \mathrm{LAG}^{+}$cells in tumor microenvironment and independently predicted better PFS and OS for patients with advanced/metastatic HCC who received anti-PD-1/anti-PD-L1-based ICI therapy. Compared with other T-cell-related signatures that were associated with response to ICI therapy, this 9-gene signature was not associated with prognosis in HCC patients who received surgery. The validity of using archival tumor tissue from different anatomic sites was also explored.

Earlier studies of exhausted CD8 signature focused on genes expressed in CD8 T cells [20] and were related to the process of $\mathrm{T}$-cell exhaustion during chronic infections $[17,36]$. Our expanded signature included additional genes associated with the dysfunctional $\mathrm{CD}^{+} \mathrm{T}$ cell in cancers. For example, CXCL13 has repetitively been found to be expressed in exhausted intra-tumoral T cells, but not in gene expression data from chronic infections [37-39]. CCL5 has also been found to be highly expressed in $\mathrm{LAG}^{+}$exhausted T cells in HCC [38], and CXCL9 reduced the $\mathrm{CD}^{+}$cytotoxic $\mathrm{T}$ cell in the tumor microenvironment of pancreatic adenocarcinoma [40]. In addition to T-cell-related genes, the expanded signature also includes genes related to other immune cells, such as macrophages (MSR1 and CSF3) and neutrophils (CYBB), reflecting the complex interaction among different immune cells in the tumor microenvironment and may help explain the better performance of our signatures in predicting response to ICI-based therapy.

Correlation between exhausted CD8 T cells in tumor microenvironment and response to ICI therapy has been extensively studied in different types of cancers [37, 4143]. Recent studies indicated that exhausted CD8 T cells consisted of $\mathrm{T}$ cells in different functional statuses, including terminally differentiated exhausted T cells and "progenitor" exhausted $\mathrm{T}$ cells that retain self-renewing properties and may be reinvigorated by ICI therapy [44]. Another extensively studied mechanism contributing to $\mathrm{T}$-cell exhaustion is aberrant vascular endothelial growth factor (VEGF) signaling and angiogenesis in the tumor microenvironment [45-47]. Combinations with other immune-modulatory agents to reinvigorate $\mathrm{T}$ cells, such as antibodies targeting the cytotoxic T-lymphocyte-associated protein 4 (CTLA-4) pathway, were also actively pursued [48]. The similar predictive value of our 9-gene exhausted CD8 T-cell signature for HCC patients who received single-agent ICI therapy or ICI-based combination therapy supported that reversal of CD8 T-cell exhaustion is a pivotal antitumor mechanism of ICI therapy.

The regulatory mechanisms of CD8 T-cell exhaustion have been extensively studied [49]. Induction and reversal of T-cell exhaustion are heavily influenced by the inflammatory and metabolic status of the tumor microenvironment $[50,51]$. The various functions represented by the genes of our 9-gene signature reflected the complex interaction in the tumor microenvironment and suggested new approaches of enhancing antitumor immunity through reversal of T-cell exhaustion. We and other investigators have found that modulation of macrophage polarization plays critical mechanistic roles in regulating CD8 T-cell exhaustion in HCC tumor microenvironment $[52,53]$. The functional role of macrophages was further supported by the association between high myeloid signature or high percentage of $\mathrm{CD} 38^{+}$macrophages in the tumors and treatment efficacy of ICI-based therapy for patients with advanced HCC $[54,55]$. Results of this kind of biomarker exploration provide rationale for more options of ICI-based combination therapy.

Compared with other signatures reflecting inflammation or immune cell function in the tumor microenvironment, the predictive value of signatures of CD8 T-cell exhaustion was least associated with prognosis of HCC patients, suggesting that they are more related to ICI therapy mechanistically. We also demonstrated good correlation between the CD8 T-cell exhaustion signature and density of $\mathrm{CD} 8{ }^{+} \mathrm{LAG}^{+}$cells in the tumors by multiplex immunofluorescence staining. Recent studies using single-cell RNA-seq approach to investigate the infiltrating $\mathrm{T}$ cells in HCC revealed that LAG3 was highly expressed in exhausted $\mathrm{T}$ cells $[56,57]$, suggesting that LAG3 might play a critical role in T-cell exhaustion in HCC. Multiplex staining of immune-related proteins in the tumor microenvironment has been found to be a valuable modality of biomarker development for immunotherapy [58]. This modality allows direct spatial localization of pertinent immune cells or markers in the tumor microenvironment $[18,59]$. Moreover, defining a cutoff value of biomarker expression based on immunofluorescence staining to identify patients who respond to treatment will be easier than using transcriptomic markers for external validation. More sophisticated technologies are evolving rapidly and may further delineate the phenotypes of exhausted CD8 T cells that best help predict efficacy of ICI therapy [60]. 
Validity of using archival tumor samples to develop immune biomarkers has been extensively discussed, and the anatomical sites of the tumor samples could be a confounding factor. Although our data indicated general similarity of immune cell contexture between primary liver and metastatic lung HCC, potential difference in specific types of immune cells or gene expression warrants further investigation. The finding of higher CD8 T-cell exhaustion signature in lung metastatic HCC was in line with our previous observation that the possibility of objective response to ICI therapy was higher for lung metastatic HCC than for liver primary/recurrent tumors [61]. This confounding factor should be considered in future biomarker research.

There are several limitations of this study. The reliability of using samples from core biopsy, which is the most common method to establish histological diagnosis of HCC, to perform transcriptomic and multiplex immunofluorescence analyses needs to be established. Analysis using core biopsy samples may be limited by sample quality and potential heterogeneity of immune microenvironment in subgroups of patients [62]. This study did not comprehensively explore the association between genetic markers (mutation loads or specific molecular aberrations) and ICI treatment efficacy. HCC is generally a cancer of low mutation burden, and the association between specific molecular aberrations and characteristics of immune microenvironment remains unclear. However, the immune-modulatory roles of common mutations in HCC, such as $\beta$-catenin and p53, and their association with tumor-intrinsic resistance to ICI therapy are increasingly recognized $[8,29,63]$. The mechanistic relationship between these molecular aberrations and CD8 $\mathrm{T}$-cell exhaustion is worthy of further investigation.

In conclusion, CD8 T-cell exhaustion markers in tumor microenvironment may predict efficacy of ICI therapy for HCC. Future clinical trials of ICI therapy for HCC should incorporate both transcriptomic and multiplex staining analyses in tumor samples to help identify patients most likely to benefit from ICI therapy.

\section{Acknowledgement}

This work was financially supported by the Centers of Genomic and Precision Medicine, National Taiwan University, Taipei, Taiwan. The authors thank the Liver Disease Prevention \& Treatment Foundation, Taiwan, for logistic support. The authors thank Taiwan Liver Cancer Network for providing the HCC tissue samples and related clinical data, the National Center for High-performance Computing for computer time and facilities as well as the second Core Lab, Department of Medical Research, National Taiwan University Hospital for providing laboratory facilities.

\section{Statement of Ethics}

This study was conducted in accordance with the mandates of the Helsinki Declaration and the guidelines of the Ethics Committee of National Taiwan University Hospital. This study was approved by the Research Ethics Committee, National Taiwan University Hospital (Approval Nos. 201308035RINC and 201706007RINA). All the patients signed written informed consent for this study.

\section{Conflict of Interest Statement}

Dr. Ann-Lii Cheng is a consultant for and a member of the speaker's bureau of Bayer-Schering Pharma. Dr. Ann-Lii Cheng is a consultant of Novartis, Merck Serono, Eisai, Merck Sharp \& Dohme (MSD) Corp., ONXEO, Bayer HealthCare Pharmaceuticals Inc., BMS Company, and Ono Pharmaceutical Co., Ltd. Dr. Ann-Lii Cheng is an associate editor of Liver Cancer. Dr. Chiun $\mathrm{Hsu}$ received research grants from BMS/ONO, Roche, and Ipsen and received honorarium from the following pharmaceutical companies: AstraZeneca, Bayer, BMS/ONO, Eisai, Eli Lilly, Ipsen, Merck Serono, MSD, Novartis, Roche, TTY Biopharm.

\section{Funding Sources}

This study was supported by the following research grants: NTU-107L9014, NTU-108L901403, NTU-109L901403 (from the Ministry of Education, Taiwan), MOST 105-2314-B-002-189MY3, MOST 105-2314-B-002-199, MOST 106-2314-B-002-229MY3, MOST 107-3017-F-002-002, MOST 107-2314-B-002-210MY3, MOST 108-2314-B-002-075-MY3, MOST 108-3017-F-002004,MOST109-2314-B-002-229-MY3,MOST109-2634-F-002-043 (from the Ministry of Science and Technology, Taiwan), YongLin Chair Grant S-01 (from National Taiwan University), NTUH107-S3837, UN108-010, UN109-051 (from National Taiwan University Hospital), Taipei Veterans General Hospital-National Taiwan University Hospital joint grant (VN-109-13).

\section{Author Contributions}

Study concept and design: D.L. Ou, C.L. Hsu, Y.M. Jeng, and C. Hsu; methodology and technical support: D.L. Ou, C.W. Chen, L. Lin, and Y.M. Jeng; provided patient sample material and clinical information: S.F. Huang and Y.M. Jeng, C. Hsu; analysis and interpretation of data: D.L. Ou, C.L. Hsu, and C. Hsu; writing, review and/or revision of the manuscript: D.L. Ou, C.L. Hsu, L.Y. Bai, S.F. Huang, A.L. Cheng, Y.M. Jeng, C. Hsu; study supervision: A.L. Cheng, Y.M. Jeng, and C. Hsu. 


\section{References}

1 Callahan MK, Postow MA, Wolchok JD. Targeting $\mathrm{T}$ cell co-receptors for cancer therapy. Immunity. 2016;44(5):1069-78.

2 Hoos A. Development of immuno-oncology drugs: from CTLA4 to PD1 to the next generations. Nat Rev Drug Discov. 2016;15(4): 235-47.

3 El-Khoueiry AB, Sangro B, Yau T, Crocenzi TS, Kudo M, Hsu C, et al. Nivolumab in patients with advanced hepatocellular carcinoma (CheckMate 040): an open-label, noncomparative, phase $1 / 2$ dose escalation and expansion trial. Lancet. 2017;389(10088): 2492-502.

4 Zhu AX, Richard SF, Julien E, Stephane C, Sadahisa O, Daniel P, et al. Pembrolizumab in patients with advanced hepatocellular carcinoma previously treated with sorafenib (KEYNOTE-224): a non-randomised, openlabel phase 2 trial. Lancet Oncol. 2018;19(7): 940-52.

5 Yau T, Park JW, Finn RS, Cheng A-L, Mathurin P, Edeline J, et al. CheckMate 459: A randomized, multi-center phase 3 study of nivolumab (NIVO) vs sorafenib (SOR) as first-line (1L) treatment in patients (pts) with advanced hepatocellular carcinoma (aHCC). Ann Oncol. 2019;30(Suppl 5):v851-v934.

6 Finn RS, Ryoo BY, Merle P, Kudo M, Bouattour M, Lim HY, et al. Results of KEYNOTE-240: phase 3 study of pembrolizumab (Pembro) vs best supportive care (BSC) for second line therapy in advanced hepatocellular carcinoma (HCC). J Clin Oncol. 2019, 37(15 Suppl 1):4004.

7 Ott PA, Bang YJ, Piha-Paul SA, Razak ARA, Bennouna J, Soria JC, et al. T-cell-inflamed gene-expression profile, programmed death ligand 1 expression, and tumor mutational burden predict efficacy in patients treated with pembrolizumab across 20 cancers: KEYNOTE-028. J Clin Oncol. 2019;37(4):318-27.

8 Kalbasi A, Ribas A. Tumour-intrinsic resistance to immune checkpoint blockade. Nat Rev Immunol. 2020;20(1):25-39.

9 Cristescu R, Mogg R, Ayers M, Albright A, Murphy E, Yearley J, et al. Pan-tumor genomic biomarkers for PD-1 checkpoint blockadebased immunotherapy. Science. 2018; 362(6411):eaar3593.

10 Calderaro J, Rousseau B, Amaddeo G, Mercey M, Charpy C, Costentin C, et al. Programmed death ligand 1 expression in hepatocellular carcinoma: relationship with clinical and pathological features. Hepatology. 2016; 64(6):2038-46

11 Dhanasekaran R, Nault JC, Roberts LR, Zucman-Rossi J. Genomic medicine and implications for hepatocellular carcinoma prevention and therapy. Gastroenterology. 2019; 156(2):492-509.
12 Sangro B, Melero I, Wadhawan S, Finn RS, Abou-Alfa GK, Cheng AL, et al. Association of inflammatory biomarkers with clinical outcomes in nivolumab-treated patients with advanced hepatocellular carcinoma. J Hepatol. 2020;73(6):1460-9.

13 Huang AC, Postow MA, Orlowski RJ, Mick R, Bengsch B, Manne S, et al. T-cell invigoration to tumour burden ratio associated with antiPD-1 response. Nature. 2017;545(7652):60-5. 14 Benci JL, Xu B, Qiu Y, Wu TJ, Dada H, Twyman-Saint Victor C, et al. Tumor interferon signaling regulates a multigenic resistance program to immune checkpoint blockade. Cell. 2016;167(6):1540-5.4e12.

15 Quigley M, Pereyra F, Nilsson B, Porichis F, Fonseca C, Eichbaum Q, et al. Transcriptional analysis of HIV-specific CD8+ T cells shows that PD-1 inhibits $\mathrm{T}$ cell function by upregulating BATF. Nat Med. 2010;16(10): 1147-51.

16 Crawford A, Angelosanto JM, Kao C, Doering TA, Odorizzi PM, Barnett BE, et al. Molecular and transcriptional basis of $\mathrm{CD} 4(+) \mathrm{T}$ cell dysfunction during chronic infection. Immunity. 2014;40(2):289-302.

17 Wherry EJ, Kurachi M. Molecular and cellular insights into T cell exhaustion. Nat Rev Immunol. 2015;15(8):486-99.

18 Thommen DS, Schumacher TN. T cell dysfunction in cancer. Cancer Cell. 2018;33(4): 547-62.

19 Robinson MD, McCarthy DJ, Smyth GK. edgeR: a bioconductor package for differential expression analysis of digital gene expression data. Bioinformatics. 2010;26(1):139-40.

20 Danaher P, Warren S, Dennis L, D'Amico L, White A, Disis ML, et al. Gene expression markers of tumor infiltrating leukocytes. J Immunother Cancer. 2017;5:18.

21 Danilova L, Wang H, Sunshine J, Kaunitz GJ, Cottrell TR, Xu H, et al. Association of PD-1/ $\mathrm{PD}-\mathrm{L}$ axis expression with cytolytic activity, mutational load, and prognosis in melanoma and other solid tumors. Proc Natl Acad Sci U S A. 2016;113(48):E7769-77.

22 Spranger S, Bao R, Gajewski TF. Melanoma-intrinsic $\beta$-catenin signalling prevents anti-tumour immunity. Nature. 2015;523(7559):231-5.

23 Ayers M, Lunceford J, Nebozhyn M, Murphy E, Loboda A, Kaufman DR, et al. IFN- $\gamma$ related $\mathrm{mRNA}$ profile predicts clinical response to PD-1 blockade. J Clin Invest. 2017; 127(8):2930-40.

24 Cabrita R, Lauss M, Sanna A, Donia M, Skaarup Larsen $M$, Mitra S, et al. Tertiary lymphoid structures improve immunotherapy and survival in melanoma. Nature. 2020; 577(7791):561-5.

25 Auslander N, Zhang G, Lee JS, Frederick DT, Miao B, Moll T, et al. Robust prediction of response to immune checkpoint blockade therapy in metastatic melanoma. Nat Med. 2018;24(10):1545-9.
26 Jiang P, Gu S, Pan D, Fu J, Sahu A, Hu X, et al. Signatures of T cell dysfunction and exclusion predict cancer immunotherapy response. Nat Med. 2018;24(10):1550-8.

27 Liu J, Lichtenberg T, Hoadley KA, Poisson LM, Lazar AJ, Cherniack AD, et al. An integrated TCGA pan-cancer clinical data resource to drive high-quality survival outcome analytics. Cell. 2018;173(2):400-16e11.

28 Thorsson V, Gibbs DL, Brown SD, Wolf D, Bortone DS, Ou Yang TH, et al. The immune landscape of cancer. Immunity. 2018;48(4): 812-30e14.

29 Harding JJ, Nandakumar S, Armenia J, Khalil DN, Albano M, Ly M, et al. Prospective genotyping of hepatocellular carcinoma: clinical implications of next-generation sequencing for matching patients to targeted and immune therapies. Clin Cancer Res. 2019;25(7): 2116-26.

30 Hale G, Liu X, Hu J, Xu Z, Che L, Solomon D, et al. Correlation of exon $3 \beta$-catenin mutations with glutamine synthetase staining patterns in hepatocellular adenoma and hepatocellular carcinoma. Mod Pathol. 2016;29(11): 1370-80.

31 Huang WJ, Tsai JH, Jeng YM. Complementary roles of $\beta$-catenin and glutamine synthetase immunostaining in diagnosis of chemotherapy-treated and untreated hepatoblastoma. J Formos Med Assoc. 2017;116(7): 549-53.

32 Luke JJ, Bao R, Sweis RF, Spranger S, Gajewski TF. WNT/ $\beta$-catenin pathway activation correlates with immune exclusion across human cancers. Clin Cancer Res. 2019;25(10): 3074-83.

33 Shimada S, Mogushi K, Akiyama Y, Furuyama T, Watanabe S, Ogura T, et al. Comprehensive molecular and immunological characterization of hepatocellular carcinoma. EBioMedicine. 2019;40:457-70.

34 Sia D, Jiao Y, Martinez-Quetglas I, Kuchuk O, Villacorta-Martin C, Castro de Moura M, et al. Identification of an immune-specific class of hepatocellular carcinoma, based on molecular features. Gastroenterology. 2017;153(3): 812-26.

35 Newman AM, Liu CL, Green MR, Gentles AJ, Feng W, Xu Y, et al. Robust enumeration of cell subsets from tissue expression profiles. Nat Methods. 2015;12(5):453-7.

36 Wherry EJ. T cell exhaustion. Nat Immunol 2011;12(6):492-9.

37 Thommen DS, Koelzer VH, Herzig P, Roller A, Trefny M, Dimeloe S, et al. A transcriptionally and functionally distinct PD-1(+) CD8(+) $\mathrm{T}$ cell pool with predictive potential in nonsmall-cell lung cancer treated with PD-1 blockade. Nat Med. 2018;24(7):994-1004.

38 Zheng C, Zheng L, Yoo JK, Guo H, Zhang Y, Guo X, et al. Landscape of infiltrating T cells in liver cancer revealed by single-cell sequencing. Cell. 2017;169(7):1342-56e16. 
39 Tirosh I, Izar B, Prakadan SM, Wadsworth $\mathrm{MH}$, Treacy D, Trombetta JJ, et al. Dissecting the multicellular ecosystem of metastatic melanoma by single-cell RNA-seq. Science. 2016; 352(6282):189-96.

40 Gao HF, Cheng CS, Tang J, Li Y, Chen H, Meng ZQ, et al. CXCL9 chemokine promotes the progression of human pancreatic adenocarcinoma through STAT3-dependent cytotoxic T lymphocyte suppression. Aging. 2020; 12(1):502-17.

41 Miller BC, Sen DR, Al Abosy R, Bi K, Virkud YV, LaFleur MW, et al. Subsets of exhausted CD8(+) T cells differentially mediate tumor control and respond to checkpoint blockade. Nat Immunol. 2019;20(3):326-36.

42 Siddiqui I, Schaeuble K, Chennupati V, Fuertes Marraco SA, Calderon-Copete S,Ferreira DP, et al. Intratumoral Tcf1(+)PD$1(+) \mathrm{CD} 8(+) \mathrm{T}$ cells with stem-like properties promote tumor control in response to vaccination and checkpoint blockade immunotherapy. Immunity. 2019;50(1):195-211.e10.

43 Yost KE, Satpathy AT, Wells DK, Qi Y, Wang C, Kageyama R, et al. Clonal replacement of tumor-specific T cells following PD-1 blockade. Nat Med. 2019;25(8):1251-9.

44 Blank CU, Haining WN, Held W, Hogan PG, Kallies A, Lugli E, et al. Defining "T cell exhaustion”. Nat Rev Immunol. 2019;19(11): 665-74.

45 Zarour HM. Reversing T-cell dysfunction and exhaustion in cancer. Clin Cancer Res. 2016; 22(8):1856-64.

46 Voron T, Colussi O, Marcheteau E, Pernot S, Nizard M, Pointet AL, et al. VEGF-A modulates expression of inhibitory checkpoints on CD8+ T cells in tumors. J Exp Med. 2015; 212(2):139-48.

47 Kim CG, Jang M, Kim Y, Leem G, Kim KH, Lee $\mathrm{H}$, et al. VEGF-A drives TOX-dependent $\mathrm{T}$ cell exhaustion in anti-PD-1-resistant mic- rosatellite stable colorectal cancers. Sci Immunol. 2019;4(41):eaay055.

48 Yau T, Kang YK, Kim TY, El-Khoueiry AB, Santoro A, Sangro B, et al. Nivolumab (NIVO) + ipilimumab (IPI) combination therapy in patients (pts) with advanced hepatocellular carcinoma (aHCC): results from CheckMate 040. J Clin Oncol. 2019;37(15 Suppl 1):4012.

49 Kallies A, Zehn D, Utzschneider DT. Precursor exhausted T cells: key to successful immunotherapy? Nat Rev Immunol. 2020;20(2): $128-36$.

50 McLane LM, Abdel-Hakeem MS, Wherry EJ. CD8 T cell exhaustion during chronic viral infection and cancer. Annu Rev Immunol. 2019;37:457-95.

51 Blank CU, Haining WN, Held W, Hogan PG Kallies A, Lugli E, et al. Defining "T cell exhaustion". Nat Rev Immunol. 2019;19(11): 665-74.

52 Liu YT, Tseng TC, Soong RS, Peng CY, Cheng $\mathrm{YH}$, Huang SF, et al. A novel spontaneous hepatocellular carcinoma mouse model for studying T-cell exhaustion in the tumor microenvironment. J Immunother Cancer. 2018;6(1):144.

53 Ou DL, Chen CW, Hsu CL, Chung CH, Feng ZR, Lee BS, et al. Regorafenib enhances antitumor immunity via inhibition of p38 kinase/ Creb1/Klf4 axis in tumor-associated macrophages. J Immunother Cancer. 2021;9(3): e001657.

54 Ng HHM, Lee RY, Goh S, Tay ISY, Lim X, Lee $\mathrm{B}$, et al. Immunohistochemical scoring of CD38 in the tumor microenvironment predicts responsiveness to anti-PD-1/PD-L1 immunotherapy in hepatocellular carcinoma. J Immunother Cancer. 2020;8(2):e000987.

55 Zhu AX, Guan Y, Abbas AR, Koeppen H, Lu $\mathrm{S}, \mathrm{Hsu} \mathrm{C}-\mathrm{H}$, et al. Genomic correlates of clinical benefits from atezolizumab combined with bevacizumab vs. atezolizumab alone in patients with advanced hepatocellular carcinoma (HCC). Cancer Res. 2020;80(Suppl 16): CT044.

56 Zheng C, Zheng L, Yoo JK, Guo H, Zhang Y, Guo X, et al. Landscape of infiltrating T cells in liver cancer revealed by single-cell sequencing. Cell. 2017;169(7):1342-56e16.

57 Liu F, Liu W, Sanin DE, Jia G, Tian M, Wang $\mathrm{H}$, et al. Heterogeneity of exhausted T cells in the tumor microenvironment is linked to patient survival following resection in hepatocellular carcinoma. Oncoimmunology. 2020; 9(1): 1746573.

$58 \mathrm{Lu}$ S, Stein JE, Rimm DL, Wang DW, Bell JM, Johnson DB, et al. Comparison of biomarker modalities for predicting response to PD-1/ PD-L1 checkpoint blockade: a systematic review and meta-analysis. JAMA Oncol. 2019; 5(8):1195-204

59 Saltz J, Gupta R, Hou L, Kurc T, Singh P, Nguyen V, et al. Spatial organization and molecular correlation of tumor-infiltrating lymphocytes using deep learning on pathology images. Cell Rep. 2018;23(1):181-93e7.

60 Parra ER, Francisco-Cruz A, Wistuba II. State-of-the-art of profiling immune contexture in the era of multiplexed staining and digital analysis to study paraffin tumor tissues. Cancers. 2019;11(2):247.

61 Lu LC, Hsu C, Shao YY, Chao Y, Yen CJ, Shih IL, et al. Differential organ-specific tumor response to immune checkpoint inhibitors in hepatocellular carcinoma. Liver Cancer. 2019;8(6):480-90.

62 Shen YC, Hsu CL, Jeng YM, Ho MC, Ho CM, Yeh CP, et al. Reliability of a single-region sample to evaluate tumor immune microenvironment in hepatocellular carcinoma. J Hepatol. 2020;72(3):489-97.

63 Munoz-Fontela C, Mandinova A, Aaronson SA, Lee SW. Emerging roles of p53 and other tumour-suppressor genes in immune regulation. Nat Rev Immunol. 2016;16(12):741-50. 